\title{
Limited and extensive cuing inversely control spatial learning performance in fornix-damaged and nonlesioned rats
}

\author{
CATHERINE MAHO and ERIC VILLEMIN \\ Laboratoire de Physiologie Nerveuse 2 \\ Centre National de la Recherche Scientifique, Gif-sur-Yvette, France \\ and \\ MARTINE AMMASSARI-TEULE \\ Istituto di Psicobiologia e Psicofarmacologia \\ Consiglio Nazionale delle Ricerche, Rome, Italy
}

\begin{abstract}
This work examines the influence of contextual manipulations on radial maze performance in fornix-damaged and control rats by presenting the task in two differentiated contexts-limited versus extensive cuing - that were successively inverted. With limited cuing, fornix-damaged rats perform worse than controls. Extensive cuing counteracts performance deficits in lesioned rats but impairs performance in controls. Inverting the contexts always disturbs performance in lesioned rats, whereas only shifting from limited to extensive cuing affects performance in controls. These results indicate that spatial learning performance of both lesioned and control rats is controlled by background stimuli, but in an opposite way.
\end{abstract}

The role of the hippocampus in selecting contextual cues to learn or to retrieve appropriate responses has been extensively studied in a number of discriminative learning tasks. Basically, these experiments have analyzed the effect of lesions to the hippocampal system on the ability of rats to organize, maintain, reverse, or transfer their responses with respect to contextual modifications.

Some discrepancies have emerged from these investigations, mainly depending on the question that is actually addressed. In fact, three main lines of researches have attempted to define the control exerted by the hippocampus on sometimes connected but fundamentally different operations: (1) paying attention to small contextual changes introduced after the animals had been previously trained, (2) using background stimuli in building up internal representations of the experimental situation during training, and (3) selecting relevant cues to guide behavior, more particularly in spatial learning tasks.

In the first case, the experiments designed to test attention toward environmental modifications in rats with hippocampal damage initially indicated that contextual changes do not modify a previously learned response. Irrelevant visual and tactile stimuli suddenly introduced in a runway failed to disturb well-established running responses in rats with extensive bilateral destruction of

Catherine Maho and Eric Villemin are affiliated with the Department of Psychophysiology, LPN2, CNRS. Correspondence should be addressed to M. Ammassari-Teule, Istituto di Psicobiologia e Psicofarmacologia, 1, Via Reno 00198 Rome, Italy (e-mail: A13900@ IRMCNR). the hippocampus (Raphelson, Isaacson, \& Douglas, 1965; Wickelgren \& Isaacson, 1963). In the same way, irrelevant auditory stimuli introduced in a leverpressing situation controlled by visual stimuli did not modify the performance of lesioned animals. However, when the contextual modification was produced by the introduction of a new stimulus related in some aspects to the stimulus controlling behavior, hippocampal animals were more distracted than controls. For example, in a task requiring animals to press two different panels sequentially at the appearance of two visual stimuli, the introduction of a third visual stimulus on a third panel profoundly disturbed the performance of hippocampus-ablated monkeys (Douglas \& Pribram, 1966).

Taken together, these results indicate deficits in attentional processes, which lead to an abnormal distractibility or indistractibility, according to the similarity of the added cue to the stimulus controlling behavior.

The problem examined in the second case concerns more directly the modalities by which hippocampusablated animals organize their responses with respect to background stimuli. Normal animals are assumed to store information concerning the whole aspects of the situation in which they are placed. According to Hirsh (1974), the hippocampus is part of a system responsible for the transfer of information from storage to performance. Since hippocampus-lesioned animals are impaired in memorizing the elements of the situation, they do not have an internal representation of the original context. Their performance, therefore, cannot rely on contextual retrieval for an accurate recall and is merely based on stimulusresponse associations correlated with the reward. 
However, this view has been challenged by experiments in which conditioning to background stimuli in hippocampus-ablated and normal animals has been specifically demonstrated. Lesions to the hippocampal system have been shown to affect the performance of rats when a single discriminative problem was successively presented in different contexts or when different discriminative problems were successively presented in the same context (Winocur \& Gilbert, 1984; Winocur \& Salzen, 1968). Moreover, the well-described impairment of hippocampus-damaged animals in reversal learning was attenuated by changing contextual conditions (Winocur \& Olds, 1978).

Finally, by using a Pavlovian conditioning procedure, Winocur, Rawlins, and Gray (1987) have shown an abnormal conditioning to context in hippocampal animals. The association, with different levels of probability, of a conditioned stimulus (CS; light, tone) to an unconditioned stimulus (US; electric shock) in a given context (black box) produced a graded aversive conditioning to the black box in control animals, inversely related to the probability that the CS predicted the US. Conversely, a near asymptotic aversive conditioning to background stimuli was observed in hippocampal animals, whether the conditioned stimulus was presented or not.

The observations reported above are of particular relevance for the question addressed in the third case: How do hippocampal lesions affect the selection of relevant stimuli to guide behavior? Indeed, an abnormal distractibility or indistractibility toward cues would necessarily affect information-gathering processes during training. In the same way, a general defect in forming appropriate internal representations of contingent events with respect to the context would delay or prevent acquisition, whatever the task considered.

In the case of spatial learning, it has been assumed that animals integrate the information gathered during exploration in a system of representations and thus acquire cognitive maps of the environment in which they move. Mapping operations have been found to depend on background stimuli. In fact, rats placed in a radial maze locate the correct arms by reference to the entire set of extra-maze cues rather than to single specific cues among those present around the maze (Diez-Chamizo, Sterio, \& Mackintosh, 1985). It seems, therefore, that providing different sets of background stimuli would lead to further insight on the mechanisms whereby normal and lesioned animals select and use contextual information in spatial learning.

In the present experiments, fornix-damaged and shamoperated rats were submitted to a radial maze task first presented in two differentiated contextual conditions-limited versus extensive cuing - that were successively inverted.

\section{METHOD}

\section{Animals}

Thirty-two male Sprague-Dawley rats weighing about 220-250 g served as subjects. They were individually housed in wire-mesh cages with food and water ad lib. Three days before pretraining began, food was removed from the cages for $12 \mathrm{~h}$. On the following day, food was individually adjusted to reduce and stabilize the weight of each rat to about $85 \%-90 \%$ of the initial level.

\section{Apparatus}

The apparatus was a wooden maze, painted gray, consisting of eight identical paths $(60 \times 12 \mathrm{~cm})$ radiating from an octagonal starting platform. An opaque plastic food cup was put at the distal end of each path. Before each trial, all the paths were baited with a 250 $\mathrm{mg}$ piece of Purina Rat Chow placed in each food cup. The maze was elevated to $60 \mathrm{~cm}$ above the floor and maintained in a constant orientation during learning.

\section{Surgery}

The rats were divided into two groups of 16 subjects each. Surgery was performed under pentobarbital anesthesia $(60 \mathrm{mg} / \mathrm{kg})$. In the first group, the dorsal fornix was partially sectioned by introducing a remote-controlled leucotome stereotaxically (posterior to bregma, $0.5 \mathrm{~mm}$; lateral from midline, $0.5 \mathrm{~mm}$; ventral from the dural surface, $5 \mathrm{~mm}$ ) and extracting the blade to a $40^{\circ}$ angle. Sham operations were performed in exactly the same way, except that the blade was not extracted from the leucotome. The animals were left in their home cages with food ad lib, for a recovery period of 1 week. At the end of Day 7, food was removed from the cages for $12 \mathrm{~h}$.

\section{Pretraining}

On Day 8 , each rat was placed on the starting platform of the apparatus and allowed to explore the paths and to consume food scattered in the whole maze. This procedure was repeated for 3 consecutive days.

\section{Training}

The training procedure started 11 days after surgery. Lesioned and sham-operated rats were divided into four subgroups (two lesioned and two sham-operated) of 8 animals each and submitted to a 10-trial learning session. On each trial, the animals were placed on the central platform and allowed to make eight choices with all the paths baited. There were 10 consecutive trials with a $24-h$ intertrial interval. Half of the subjects (8 lesioned and 8 sham-operated) learned the radial-eight-arm-maze problem with limited visual cuing. The maze was placed in an empty room with white walls uniformly illuminated. The other subjects learned the task with extensive visual cuing. In this case, the maze was placed in a room containing numerous objects and panels with multioriented stripes but without redundant stimuli (see Figure 1). Four dependent variables were recorded: the number of correct paths nun before the first error, the number of correct choices out of eight, the time spent in running the maze, and the degree of divergence of the strategies. This last parameter, introduced by Lachman and Brown (1957), is obtained as follows: Within a trial, all the transitions between two arms run consecutively are evaluated by counting one unit of divergence when the rats run two adjacent arms, by counting two units of divergence when the rats run two arms separated by one arm, by counting three units of divergence when the rats run two arms separated by two arms, and so on. For the whole sequence of runs displayed within the trial, the units of divergence corresponding to each transition are summed up and give the final value - that is, the degree of divergence of the maze-running strategy. For example, for the errorless sequence of runs 1-2-4-5-3-6-7-8, the degree of divergence will be obtained by summing up the following units of divergence: $1+2+1+2+3+1+1$, which give the number 11 . It must be noted that the degree of divergence of a clockwise or a counterclockwise strategy is 7 and that 16 different patterns correspond to this category. Since in this experiment the number of runs within a trial was constant (8), we calculated the degree of divergence of each pattern of choice given by each animal on each trial, errorless or not. 




Figure 1. Schematic representation of the overloaded context: (1) Table with a yellow washing tray and numerous objects, (2) chair of the experimenter, (3) cardboard panel $(2 \times 2 \mathrm{~m})$ with black and white squares, (4) shelves with empty cages, (5) black cardboard panel $(60 \times 120 \mathrm{~cm}),(6)$ metal panel with black and white stripes, (7) electrophysiological recordings material, (8) white sink, (9) windows with black curtains, and (10) window without curtains. Two lamps are on the west side; daylight is on the east side.

\section{Contextual Reversal}

On the day after the 10th learning trial, the groups were transferred from one context to the other, and their performance was examined for two daily trials.

\section{Histology}

At the end of testing, the rats were deeply anesthetized with pentobarbital $(80 \mathrm{mg} / \mathrm{kg})$ and perfused intracardially with saline followed by formalin (10\% solution). The brains were fixed in this solution for 1 day, sectioned sagittally $(60 \mu \mathrm{m})$, and stained with cresyl violet. Six control and lesioned brains from a pilot experiment were perfused with buffered formalin $(\mathrm{pH}: 7,4)$ and sectioned coronally for AChE staining.

\section{Statistics}

The training data were statistically evaluated by a three-factor between-within analysis of variance, the between factors being lesion (two levels) and cuing condition (two levels), the within factor being trials (10 levels). Post hoc between-group comparisons were made by a contrast analysis. The data concerning contextual reversal were analyzed in each group by $t$ tests for paired samples plotted against the scores from the last training trial and those from the first reversal trial.

\section{RESULTS}

\section{Histology}

Examination of the tissue revealed a partial dorsal fornix section involving about half of the fibers projecting from the septum onto the hippocampus (Figure 2). Coronal section stained for AChE indicated a normal AChE staining in sham-operated rats (Figure $3 \mathrm{~A}$ ). AChE depletion, slightly asymmetric, was observed in fornixdamaged rats (Figure 3B).

\section{Training}

A significant effect of the trials for the number of correct choices out of eight within each trial $[F(9,252)=$ $11.87, p<.001]$ and the number of correct paths run before the first error $[F(9,252)=15.93, p<.001]$ showed that choice accuracy improved with training in all groups (Figures $4 \mathrm{~A}$ and $4 \mathrm{~B}$ ). However, no main effect of the lesion or of the cuing condition, but a significant effect of the lesion $\times$ cuing condition interaction for both variables $[F(1,28)=8.54, p<.006 ; F(1,28)=$ $6.76, p<.014]$, indicated that the characteristics of the context exert a different control on spatial learning performance, depending on whether the animals were lesioned or not. In fact, between-group comparisons pointed out that (1) fornix-damaged rats performed better than sham-operated rats with extensive cuing $[F(1,14)=5.64$, $p<.05 ; F(1,14)=4.74, p<.05],(2)$ fornix-damaged rats performed worse than sham-operated rats with limited cuing $[F(1,14)=6.74, p<.01 ; F(1,14)=5.36$, $p<.05$ ], and (3) the performance of sham-operated rats with extensive cuing was significantly lower than that of sham-operated rats with limited cuing $[F(1,14)=9.75$, $p<.01 ; F(1,14)=5.37, p<.05]$.

The data concerning the degree of divergence of the maze-running strategies (Figure $4 \mathrm{C}$ ) also revealed a significant effect of the trials $[F(9,252)=2.11, p<.05]$. This indicates that animals from all groups optimized their patterns of choice in collecting food, since they tended to choose more proximate arms as learning proceeded. However, this behavior was particularly evident in shamoperated animals with limited cuing that displayed patterns of choice significantly less divergent than those observed in the three other groups $[F(1,28)=4.92$, $p<.05]$. No influence of the contextual characteristics on this parameter was evident in lesioned animals.

Finally, the time spent in running the maze also decreased with practice in all groups [significant effect of the trials, $F(9,252)=14.60, p<.001]$, but, throughout training, sham-operated rats with extensive cuing ran the maze significantly slower $[F(1,14)=4.86, p<.05]$ than sham-operated rats with limited cuing (Figure 4D).

\section{Contextual Reversal}

The transfer from the extensive- to the limited-cuing condition had no effect on the performance or on the maze-running strategies of nonlesioned rats. In fornixdamaged rats, however, the suppression of extra-maze cues significantly reduced the number of correct choices $(t=2.64, p=.016)$. Conversely, the transfer from the limited- to the extensive-cuing condition disturbed the performance of both groups, the nonlesioned rats being more impaired than the fornix-damaged rats. The addition of 




Figure 2. Sagittal section stained with cresyl violet, representative of the dorsal fornix section.

extra-maze cues significantly reduced the number of correct choices in nonlesioned $(t=2.64, p=.016)$, as well as in fornix-damaged ( $t=2.04, p=.038$ ) rats, whereas the first error occurred significantly earlier in nonlesioned rats only $(t=2.12, p=.012)$. These performance alterations disappeared on the second reversal trial (Figures $4 A$ and $4 B)$.

\section{DISCUSSION}

The results show that, with extensive cuing, fornixdamaged rats perform better than sham-operated rats. It seems, therefore, that this contextual condition induces distractibility in the latter group, probably because the animals are engaged in encoding numerous extra-maze visual stimuli. This hypothesis is consistent with the fact that they spent significantly more time in running the maze than did sham-operated rats placed in the limited-cuing condition. In addition, they were also impaired in the organization of maze-running strategies. With training, there was no decrease in the degree of divergence of their patterns of choices, whereas in the other groups, there was a consistent trend to run more proximate arms. It seems, therefore, that in the nonlesioned group, paying attention to a large number of visual extra-maze cues produces interference instead of facilitating orientation and location of correct arms in the radial maze.

The high performance of fornix-damaged rats in the extensive-cuing condition suggests that these animals are less distractible than controls, probably because they are impaired in processing visual contextual cues (Samuels, 1972). Nevertheless, fornix-damaged rats seem to use some cues for orientation in the maze, since they perform significantly better in the extensive- than in the limitedcuing condition. At first, these results are in agreement with previous data showing that cuing improves memory performance in animals with damage to the hippocampal system (Ellen \& Deloache, 1968; Winocur \& Olds, 1978). However, in these experiments, cuing was found to increase performance in lesioned subjects but not to decrease performance in controls. In the present experiment, a correct orientation in an extensively cued context requires complex operations of stimulus selection that, paradoxically, fornix-damaged, but not sham-operated, rats were able to perform. It seems, therefore, that partially cutting the fornix reduces the processing of background stimuli, probably through a general decline of attention. This reduction would improve performance in lesioned 

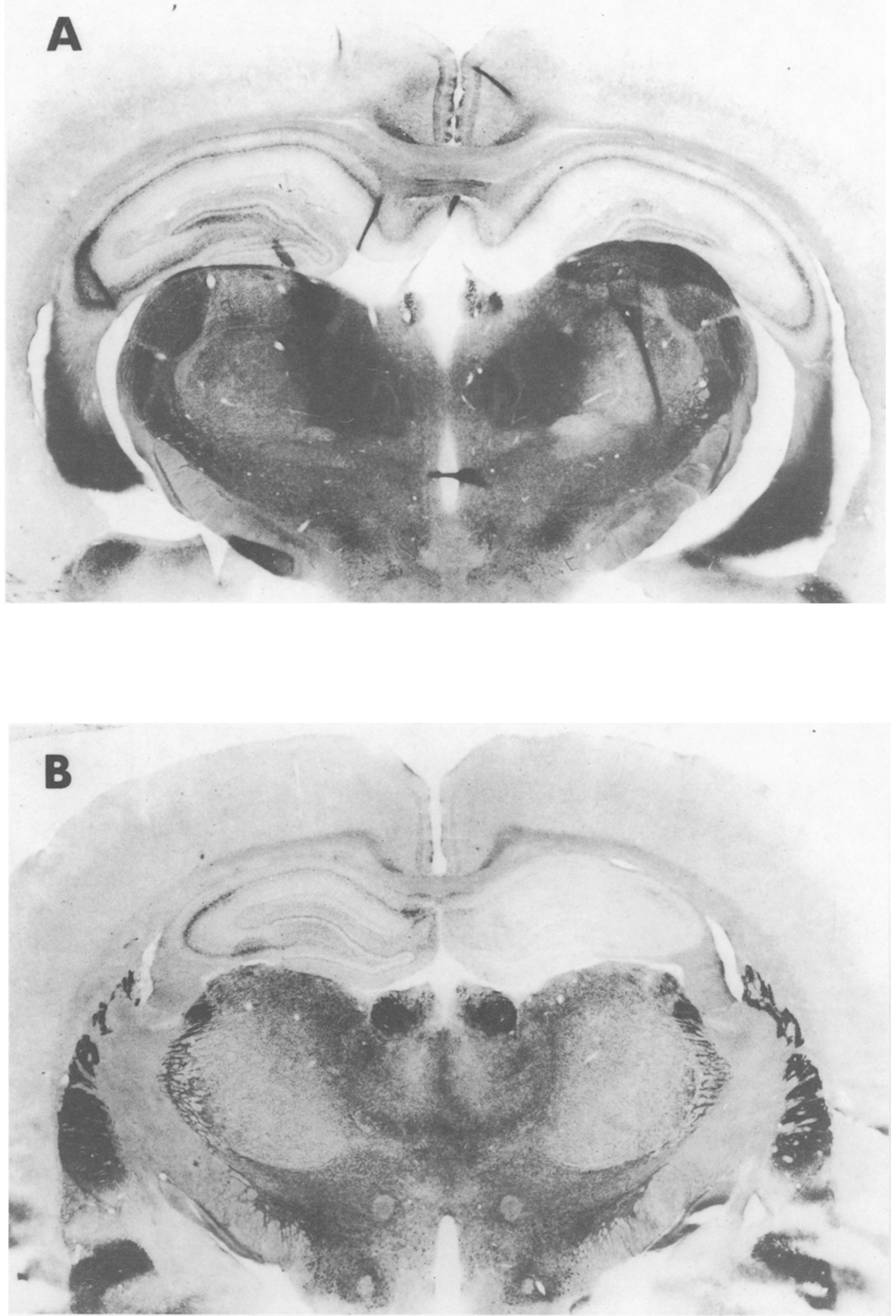

Figure 3. Coronal sections showing hippocampal AChE staining in (A) control and (B) fornix-damaged rats. 





Figure 4. Learning scores, degrees of divergence of the strategies, and maze-running times during acquisition and contextual reversal. (A) Correct choices out of eight. (B) Correct paths run before the first error. (C) Degrees of divergence of the strategies. (D) Maze-running times with SE. L-LC, lesioned rats with limited cuing; L-EC, lesioned rats with extensive cuing; S-LC, sham-operated rats with limited cuing; S-EC, sham-operated rats with extensive cuing. Time is expressed in seconds. 
rats, allowing them (1) to ignore part of the elements present in the extensively cued context and (2) to use the most salient cues to organize their sequences of runs.

Thus, in this experiment, high learning scores are observed in two groups: lesioned animals with extensive cuing and sham-operated animals with limited cuing. Quantitative parameters indicated that these animals had similar performances. Nevertheless, differences were found in the organization of their maze-running strategies. Rats from both groups tended to optimize their sequences of choices in collecting food, since the degree of divergence of their pattern of choice decreased throughout learning. However, this behavior was significantly more marked in sham-operated rats placed in the limited cuing condition. It seems, therefore, that in spite of their high learning scores, some defects in the organization of spatial behavior persist in fornix-damaged rats placed in the extensive cuing condition. This observation is consistent with evidence indicating that performance deficits following damages to the hippocampal system could be selectively spatial (Nadel \& MacDonald, 1980).

The well-described impairment of spatial learning in fornix-damaged rats relative to sham-operated rats was exclusively found with limited cuing. Indeed, some cues were still present in the latter situation, but they appeared insufficient to support a correct orientation in the radial maze. Thus, if extensive cuing can counteract performance deficits in lesioned rats, the main effect of the lesion cannot be ascribed to a mnemonic or a cognitive impairment. The low performance of lesioned rats with limited cuing rather reflects an inability to select or to encode relevant cues among those present in the limitedcuing context (Rickert, Lorden, Dawson, Smyly, \& Callaghan, 1979; Winocur \& Salzen, 1968).

Interestingly, nonlesioned animals with extensive cuing performed the same as lesioned animals with limited cuing, thus suggesting that the multiplicity of extra-maze cues can impair spatial learning performance to the same extent as the lesion itself.

Finally, the results concerning the transfer from one context to the other indicate that suppressing cues-that is, shifting from extensive to limited cuing-did not modify performance in sham-operated rats but decreased learning scores in fornix-damaged rats. This observation would suggest that the performance of lesioned rats mainly relies on the use of extra-maze cues. However, adding cues-that is, shifting from limited to extensive cuingdecreased choice accuracy in both groups on the first reversal trial, with the main impairing effect observed in the sham-operated group. Fornix-damaged rats, therefore, showed a higher reactivity to change, since their performance was impaired by the suppression, as well as the addition, of extra-maze cues. This result is consistent with the finding that rats with damage to the hippocampal system are more constrained by contextual modifications than are nonlesioned rats (Winocur \& Gilbert, 1984). Conversely, performance deficits in sham-operated rats were observed only when the number of background stimuli was increased, thus suggesting that the added stimuli induced interference. Nevertheless, the disturbing effect of the extensively cued context was transient, since on the second reversal trial, choice accuracy improved in both groups.

Indeed, the present experimental situations differ widely from the discriminative paradigms that are generally used to investigate the role played by contextual stimuli in learning and memory processes. Nevertheless, some conclusions from the present findings agree with previous data concerning the behavior of rats with lesions to the hippocampal system. Fornix-damaged animals process and/or encode less extra-maze stimuli (Winocur \& Gilbert, 1984), exhibit attentional-like deficits (Rickert et al., 1979), perform better when the salience of cues is increased (Ellen \& Deloache, 1968), display less adaptive maze-running strategies (Ammassari-Teule \& Maho, 1985; Maho, Dutrieux, \& Ammassari-Teule, 1988), and are more influenced by contextual changes (Winocur \& Gilbert, 1984). However, fornix-damaged rats are able to correctly perform the radial maze task in an extensively cued context in which they develop a paradoxical selective attention toward background stimuli. Finally, the present experiments also show that manipulating the number of contextual elements can affect spatial learning in nonlesioned rats. In particular, the same extensive-cuing condition counteracts performance deficits in fornix-damaged rats but produces, in nonlesioned rats, learning impairments equivalent to those induced by the lesion in the limited-cuing condition. This observation suggests that the control exerted by specific brain structures on spatial learning performance is modulated by environmental factors.

\section{REFERENCES}

Ammassarj-Teule, M., \& Maho, C. (1985). Properties of mapping induced by fornix damages: Leaming and memorizing the radial maze task. Physiological Psychology, 13, 230-234.

Diez-Chamizo, V., Steruo, D., a Mackuntosh, N. J. (1985). Blocking and overshadowing between intra-maze and extra-maze cues: $A$ test of the independence of locale and guidance learning. Quarterly Journal of Experimental Psychology, 37, 235-253.

Douglas, R. J., Pribram, K. H. (1966). Learning and limbic lesions. Neuropsychologia, 4, 197-220.

Ellen, P., Deloache, J. (1968). Hippocampal lesions and spontaneous alternation behavior in the rat. Physiology \& Behavior, 3, 857-860.

Hirsh, R. (1974). The hippocampus and contextual retrieval of information from memory: A theory. Behavioral Biology, 12, 421-444.

Lachman, S. J., Brown, C. R. (1957). Behavior in a free choice multiple path elimination problem. Journal of Psychology, 13, 101-109.

Maho, C., Dutrieux, G., \& Ammassaru-Teule, M. (1988). Parallel modifications of spatial learning performances, exploration pattems, and hippocampal theta rhythms in fornix-damaged rats: Reversal by oxotremorine. Behavioral Neuroscience, 102, 601-604.

Nadel, L., \&acDonald, L. (1980). Hippocampus: Cognitive map or working memory? Behavioral \& Neural Biology, 29, 405-409.

Raphelson, A. C., Isaacson, R. L., Douglas, R. J. (1965). The effect of distracting stimuli on the runway performance of limbic damaged rats. Psychonomic Science, 3, 483-484.

Rickert, E. J., Lorden, J. F.; Dawson, R., JR., Smyly, E., \& Callaghan, M. F. (1979). Stimulus processing and stimulus selec- 
tion in rats with hippocampal lesions. Behavioral \& Neural Biology, 27, 454-465.

SAmUELS, I. (1972). Hippocampal lesions in the rat: Effects on spatial and visual habits. Physiology \& Behavior, 8, 1093-1098.

Wickelgren, W. O., IsAacson, R. L. (1963). Effect of the introduction of an irrelevant stimulus upon runway performance of the hippocampectomized rat. Nature, 200, 48-50.

Winocur, G., GilberT, M. (1984). The hippocampus, context, and information processing. Behavioral \& Neural Biology, 40, 27-43.

Winocur, G., OLDS, J. (1978). Effects of context manipulation on memory and reversal learning in rats with hippocampal lesions. Joumal of Comparative \& Physiological Psychology, 92, 312-321.
Winocur, G., Rawlins, J. N. P., Gray, J. A. (1987). The hippocampus and conditioning to contextual cues. Behavioral Neuroscience, 101, 617-625.

Winocur, G., SALZEN, E. A. (1968). Hippocampal lesions and transfer behavior in the rat. Journal of Comparative \& Physiological Psychology, 65, 303-310.

(Manuscript received February 20, 1991: revision accepted for publication September 10, 1991.)

\section{Announcement}

\section{(C)}

DISCIPLINES FOR THE 1992 COMPETITION

- NATURAL SCIENCES

- SOCIAL SCIENCES

- ACCOUNTING

Deadline for the

1992 Competition is

February 24, 1992
SIXTH ANNUAL EDUCOM HIGHER EDUCATION SOFTWARE AWARDS COMPETITION

The EDUCOM Higher Education Software Awards Program was established in 1987 to recognize and promote quality educational software and computer-based teaching innovations in higher education. The program has two divisions:

- Product Division, for original software.

- Curriculum Innovation Divislon, for the innovative uses of new or existing software to solve important instructional problems.

Entry Forms: For entry forms and further information please contact:

Higher Education Software Awards Program, attn: Gail Miller Computer Science Center, Bullding 224

University of Maryland

College Park, Maryland 20742-2411

(301) 405-7534 E-mail: awards@cristal.umd.edu

Charter Sponsors:

Apple Computer, Inc.• IBM Corp. • NeXT Computer

Contributing Sponsors:

Bell Atlantic Corp., Claris Corp., Microsoft Corp., National Center for Automated Information Retrieval (NCAIR), Sun Microsystems.

Other Sponsors: EDUCOM, The University of Maryland 